\title{
On hybrid temporal basis functions for stable numerical solution of time domain boundary integral equations
}

Fang Q. Hu

Correspondence: fhu@odu.edu Department of Mathematics and Statistics, Old Dominion University, Norfolk, Virginia, USA

\begin{abstract}
Problems in unsteady aerodynamics and aeroacoustics can sometimes be formulated as integral equations, such as the boundary integral equations. Numerical discretization of integral equations in the time domain often leads to so-called March-On-in-Time (MOT) schemes. In the literature, the temporal basis functions used in MOT schemes have been largely limited to low-order shifted Lagrange basis functions. In order to evaluate the accuracy and effectiveness of the temporal basis functions, a Fourier analysis of the temporal interpolation schemes is carried out. Based on the Fourier analysis, the spectral resolutions of various temporal basis functions are quantified. It is argued that hybrid temporal basis functions be used for interpolation of the numerical solution and its derivatives with respect to time. Stability of the proposed hybrid schemes is studied by a matrix eigenvalue method. Substantial improvement in accuracy and efficiency by using the hybrid temporal basis functions for time domain integral equations is demonstrated by numerical examples. Compared with the traditional temporal basis functions, the use of hybrid basis functions keeps numerical errors low for a larger frequency range given the same time step size. Conversely, for a given range of frequency of interest, a larger time step can be used with the hybrid temporal basis functions, resulting in an increase in computational efficiency and, at the same time, a reduction in memory requirement.
\end{abstract}

Keywords: Time domain integral equation, Temporal basis function, March-On-in-Time

\section{Introduction}

Unsteady aerodynamics and aeroacoustics problems can sometimes be formulated as integral equations. For instances, boundary integral equations can be derived for the quasi-potential flows and for acoustic wave scattering problems [1-8]. Historically, numerical solutions of integral equations are often carried out in the frequency domain. A vast majority of computational solvers for boundary integral equations have been developed assuming a single fixed frequency. Compared with the frequency domain approach, time domain solutions have several distinct advantages. For instances, solutions at all frequencies can be obtained within one single computation by post-processing the time domain solution, and acoustic sources of broadband nature can be more naturally simulated in the time domain. However, the development of time domain approach has been hindered by two major difficulties. The first was the intrinsic instability in the time

(c) The Author(s). 2019 Open Access This article is distributed under the terms of the Creative Commons Attribution 4.0 International License (http://creativecommons.org/licenses/by/4.0/), which permits unrestricted use, distribution, and reproduction in any medium, provided you give appropriate credit to the original author(s) and the source, provide a link to the Creative Commons license, and indicate if changes were made. 
domain formulations due to the presence of resonant frequencies which are bound to exist in time domain calculations. The second was the formidably high computational costs in solving the time domain integral equation. Significant progresses have been made in recent years in resolving these two major difficulties. Stable boundary integral formulation can now be achieved by using a Burton-Miller type reformulation or a discretization by Convolution Quadrature [1, 3, 5, 8-10]. And there are recent developments where computational complexity can be reduced from $O\left(N_{t} N^{2}\right)$ to $O\left(N_{t} N \log ^{2} N\right)$ where $N$ is the total number of unknowns on the scattering surface in the three-dimensional space, and $N_{t}$ is the total number of time steps $[11,12]$. For instance, for March-On-in-Time (MOT) schemes, the recently developed Plane Wave Time Domain (PWTD) algorithm can be used to accelerate the far interactions [11], akin to the Fast Multipole Methods in the frequency domain. Another efficient time domain propagation algorithm is the multi-level Cartesian Non-uniform Grid Time Domain Algorithm (CNGTDA), based on the delay- and amplitude-compensated acoustic field [12-14]. With these breakthroughs, as well as the advances in computational power and new computing architectures, an increase in the application of time domain integral equations for unsteady aerodynamics and aeroacoustics is expected in the future.

Compared to these recent efforts, far less attention has been given to the temporal basis functions used in the MOT schemes. In fact, with a few exceptions (e.g., [15-19]), the time basis functions used in MOT schemes are still largely limited to low-order shifted Lagrange basis functions. The focus of the present paper is on suitable temporal basis functions for the solution of time domain integral equations. Improving temporal basis functions is important because it will lead to larger allowable time step sizes and will, in turn, significantly reduce the total number of time steps $N_{t}$, and thus has a direct impact on the efficiency and accuracy as well as memory requirements of a time domain solver. Currently, the most popular temporal basis function remains to be the third-order shifted Lagrange polynomial. A traditional metric of assessing the accuracy of basis functions has been the order of the polynomials employed. In the present paper, we study the accuracy in the Fourier frequency space. Unlike the traditional Taylor series analysis on the temporal basis functions, a Fourier analysis examines the error in the Fourier frequency space. Lagrange basis functions up to the 6th-order are analyzed for their spectral resolution. As a result of the Fourier analysis, it is shown that for a given order of the basis functions, the spectral resolutions for approximating the solution are different from that for approximating its temporal derivatives that are also required in the integral equation formulation. To improve the overall temporal spectral resolution, it is proposed that hybrid temporal basis functions be used in the MOT schemes where different basis functions are used for interpolating the solution and it derivatives. Stability of the proposed hybrid schemes is validated by a numerical matrix eigenvalue analysis.

The rest of the paper is organized as follows. In Section 2, formulation of a time domain integral equation is introduced as it appears in acoustic scattering problems. The need for temporal basis functions is demonstrated when the integral equation is discretized as a March-On-in-Time scheme. In Section 3, the Lagrange basis function is defined and formulated as an interpolation problem. Its essential properties as an interpolation tool are discussed. In Section 4, a Fourier analysis of the Lagrange basis functions is presented. Based on the Fourier analysis, hybrid schemes are proposed that increase the spectral resolution of the interpolation in the numerical solution of integral equations. Stability 
of the proposed hybrid schemes for the solution of time domain integral equation is discussed in Section 5. Numerical examples that demonstrate the effectiveness of the hybrid schemes are presented in Section 6. Section 7 has the conclusions.

\section{Time domain boundary integral equations and temporal basis functions}

As an example of time domain integral equation and its requirements on the temporal basis functions developed in the present paper, we consider the problem of acoustic scattering which, under the no-flow assumption, is governed by the wave equation with a source term,

$$
\frac{\partial^{2} u}{\partial t^{2}}-c^{2} \nabla^{2} u=q(\boldsymbol{r}, t)
$$

The domain $V \in \mathbb{R}^{3}$ is exterior of a scattering body defined by a closed surface, or surfaces, $S$. In (1), $c$ is the wave speed and $q$ is the known source. The wave equation can be converted into a Time Domain Boundary Integral Equation (TDBIE) as follows (e.g., $[3,5,6,8])$ :

$2 \pi u\left(\overline{\boldsymbol{r}}_{s}, t\right)=\frac{1}{c^{2}} \int_{V} \frac{1}{R} q\left(\boldsymbol{r}, t_{R}\right) d \boldsymbol{r}+\int_{S} \frac{1}{R}\left[\frac{\partial u}{\partial n}\left(\boldsymbol{r}_{s}, t_{R}\right)+\frac{1}{R} \frac{\partial R}{\partial n} u\left(\boldsymbol{r}_{s}, t_{R}\right)+\frac{1}{c} \frac{\partial R}{\partial n} \frac{\partial u}{\partial t}\left(\boldsymbol{r}_{s}, t_{R}\right)\right] d \boldsymbol{r}_{s}$

where $\overline{\boldsymbol{r}}_{S}$ is a smooth point on surface $S, \frac{\partial}{\partial n}$ is the in-ward normal derivative on $S$, and $t_{R}$ is the retarded time between $\overline{\boldsymbol{r}}_{s}$ and $\boldsymbol{r}_{s}$ :

$$
t_{R}=t-R / c, \quad \text { where } R=\left|\overline{\boldsymbol{r}}_{s}-\boldsymbol{r}_{s}\right|
$$

For a boundary value problem, the normal derivative $\partial u / \partial n$ is often given and therefore Eq. (2) establishes an integral equation for the solution $u\left(\boldsymbol{r}_{s}, t\right)$ on the scattering surface. For instance, if $u$ represents acoustic velocity potential, then on solid surfaces, we have $\partial u / \partial n=0$. To solve the integral Eq. (2) by a Time Domain Boundary Element Method (TDBEM), the integral surface $S$ is discretized into boundary elements $S_{i}$, i.e., $S=\bigcup S_{i}$, and the time $t$ is discretized by a temporal grid with a uniform time step $\Delta t$,

$$
t_{j}=t_{0}+j \Delta t
$$

where $t_{0}$ is an arbitrary time at $j=0$. Numerical solution for $u\left(\boldsymbol{r}_{s}, t\right)$, denoted by $U\left(\boldsymbol{r}_{s}, t\right)$, is often conveniently expressed as an expansion in surface and temporal basis functions as

$$
U\left(\boldsymbol{r}_{s}, t\right)=\sum_{i=1}^{N_{e}} \sum_{j=0}^{N_{t}} u_{i}^{j} \psi_{j}(t) \varphi_{i}\left(\boldsymbol{r}_{s}\right)
$$

in which $N_{e}$ is the total number of surface nodes and $N_{t}$ is the total number of time steps. In this expression, $\varphi_{i}\left(\boldsymbol{r}_{s}\right)$ is the surface basis function for the $i$-th surface node, and $\psi_{j}(t)$ denotes the temporal nodal basis function for $t_{j}$. For simplicity, a constant boundary element approach is assumed in this paper where $\varphi_{i}\left(\boldsymbol{r}_{s}\right)$ is unity when $\boldsymbol{r}_{s}$ is inside the $i$-th element and is zero elsewhere. Then, the expansion coefficients in (5), $u_{i}^{j}$, represent the solution at the $i$-th node and at time $t_{j}$. Furthermore, in this paper, only temporal basis functions with a finite support are considered. Specifically, we assume $\psi_{j}(t)=0$ for $t \leq t_{j-1}$ or $t>t_{j+m}$ where $m$ is a fixed constant. Consequently, the summation over index $j$ in (5) will result in no more than $m+1$ non-zero terms for any given $t$.

In the collocation MOT approach, expansion (5) is substituted into (2) and the integral equation is enforced at each surface nodal collocation point and progressively at each 
time step. Using the discretization stipulated in (5), the MOT approach for Eq. (2) results in an algebraic iteration scheme of the form

$$
\boldsymbol{B}_{0} \boldsymbol{u}^{n}=\boldsymbol{q}^{n}-\sum_{k=1}^{K} \boldsymbol{B}_{k} \boldsymbol{u}^{n-k}
$$

where $\boldsymbol{u}^{n}$ denotes the vector of all coefficients $u_{i}^{n}$ at time level $t_{n}, \boldsymbol{q}^{n}$ denotes the source term generated by the volume integral in (2), and $K$ denotes the limit for index $k$. The matrices $\boldsymbol{B}_{0}, \boldsymbol{B}_{1}, \ldots, \boldsymbol{B}_{K}$ are the coefficient matrices resulting from the discretization of TDBIE (2) as described above. Explicit expressions for the $\boldsymbol{B}$ matrices can be found in [8].

While it has been well-known that the MOT scheme derived directly from the TDBIE (2) suffers from long time instability, the instability can be effectively eliminated by applying a Burton-Miller type reformulation to the TDBIE $[1,3,5,8]$. The Burton-Miller reformulation involves applying the follow differential operator, a linear combination of the time and normal derivatives, to the TDBIE (2):

$$
\frac{\partial}{\partial t}-c \frac{\partial}{\partial n}
$$

As evident from the discussions above, to solve the integral Eq. (2), there is a need to evaluate the solution $U\left(\boldsymbol{r}_{s}, t\right)$ and its temporal derivative $\frac{d U}{d t}\left(\boldsymbol{r}_{s}, t\right)$ at retarded time $t_{R}=t_{n}-R / c$ which may or may not fall on temporal nodal points. When $t_{R}$ does not lie on a nodal point, approximation by interpolation would be required. Furthermore, when Eq. (2) is cast into a Burton-Miller type reformulation by the operator (7), there is also a need to compute the second-order time derivative $\frac{d^{2} U}{d t^{2}}\left(\boldsymbol{r}_{s}, t\right)$. It has been customary in the literature that these time derivatives are to be computed using the same temporal basis functions as in expansion (5). This results in the following approximations:

$$
\begin{aligned}
& \frac{\partial U}{\partial t}\left(\boldsymbol{r}_{s}, t\right)=\sum_{i=1}^{N_{e}} \sum_{j=0}^{N_{t}} u_{i}^{j} \psi_{j}^{\prime}(t) \varphi_{i}\left(\boldsymbol{r}_{s}\right) \\
& \frac{\partial^{2} U}{\partial t^{2}}\left(\boldsymbol{r}_{s}, t\right)=\sum_{i=1}^{N_{e}} \sum_{j=0}^{N_{t}} u_{i}^{j} \psi_{j}^{\prime \prime}(t) \varphi_{i}\left(\boldsymbol{r}_{s}\right)
\end{aligned}
$$

where $\psi_{j}^{\prime}(t)$ and $\psi_{j}^{\prime \prime}(t)$ denote, respectively, the first and second derivatives of $\psi_{j}(t)$ with respect to $t$.

The focus of the present paper is to study in detail suitable temporal basis functions $\psi_{j}(t)$ to be used for computing $U\left(\boldsymbol{r}_{s}, t\right), \frac{\partial U}{\partial t}\left(\boldsymbol{r}_{s}, t\right)$, and $\frac{\partial^{2} U}{\partial t^{2}}\left(\boldsymbol{r}_{s}, t\right)$.

\section{Formulation of temporal basis functions}

\subsection{Classical shifted Lagrange basis function}

In the literature (e.g., $[3,5,16])$, temporal basis function $\psi_{j}(t)$, appearing in $(5)$, is often taken to be of the following form,

$$
\psi_{j}(t)=\Psi(\tau)=\left\{\begin{array}{cc}
\Psi_{0}(\tau) & -1<\tau \leq 0 \\
\Psi_{-1}(\tau) & 0<\tau \leq 1 \\
\Psi_{-2}(\tau) & 1<\tau \leq 2 \\
\ldots & \ldots \\
\Psi_{-m}(\tau) & m-1<\tau \leq m \\
0 & \text { other }
\end{array}\right.
$$


in which $\tau=\left(t-t_{j}\right) / \Delta t, m$ is the chosen order of basis function, and $\Psi_{\ell}(\tau), \ell=-m, \ldots, 0$, are shifted Lagrange polynomials formed as follows:

$$
\Psi_{\ell}(\tau)=\frac{\prod_{i=-m, i \neq \ell}^{0}[-\tau+(i-\ell)]}{\prod_{i=-m, i \neq \ell}^{0}(i-\ell)}, \quad \ell=-m, \ldots, 0
$$

Here, the product notation is used where, e.g., $\prod_{i=1}^{n} a_{i}$ denotes $a_{1} \cdot a_{2} \cdot a_{3} \cdots a_{n}$. For convenience of discussion, $\Psi_{\ell}(\tau)$ will be referred to as $\tau$-normalized basis functions. Equation (11) yields a temporal basis function that is a piece-wise $m$-th order polynomial. For instances, the popular third-order shifted Lagrange basis functions are the following:

$$
\Psi(\tau)=\left\{\begin{array}{cc}
1+\frac{11}{6} \tau+\tau^{2}+\frac{1}{6} \tau^{3} & -1<\tau \leq 0 \\
1+\frac{1}{2} \tau-\tau^{2}-\frac{1}{2} \tau^{3} & 0<\tau \leq 1 \\
1-\frac{1}{2} \tau-\tau^{2}+\frac{1}{2} \tau^{3} & 1<\tau \leq 2 \\
1-\frac{11}{6} \tau+\tau^{2}-\frac{1}{6} \tau^{3} & 2<\tau \leq 3 \\
0 & \text { other }
\end{array}\right.
$$

Function $\Psi(\tau)$ is plotted in Fig. 1 for cases of $m=3,4,5$, and 6. It represents the influence of the nodal value at $t_{j}$ on other points in time. Here, the temporal basis function is considered to be causal on a discretized grid if $\psi_{j}(t)=0$ for all $t \leq t_{j-1}$. Because the
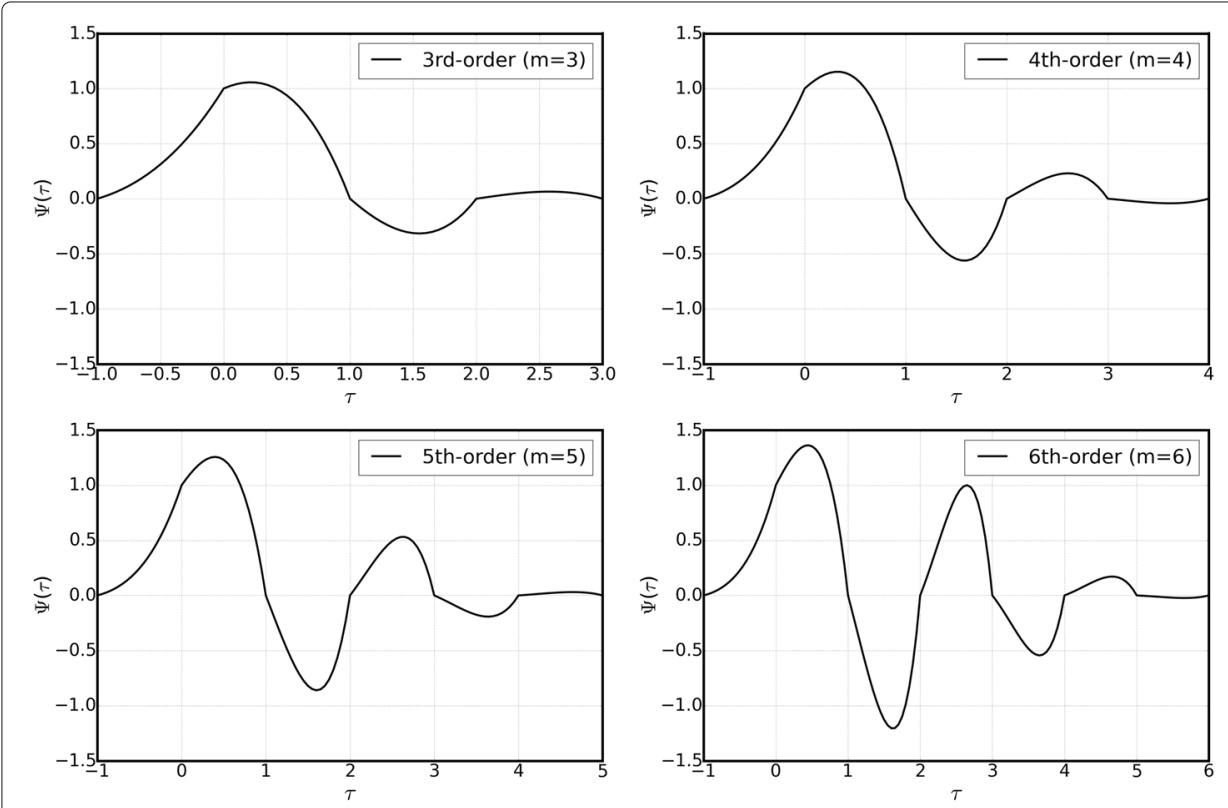

Fig. $1 \tau$-normalized basis function for the 3rd- to 6-th order Lagrange basis functions as defined in (11). Here $\tau=\left(t-t_{j}\right) / \Delta t$ with respect to temporal node $t_{j}$ 
temporal basis function (10) has a finite support in time and causal by design, we have, for any $t \in\left(t_{n-1}, t_{n}\right]$,

$$
\sum_{j=0}^{N_{t}} u_{i}^{j} \psi_{j}(t)=u_{i}^{n} \psi_{n}(t)+u_{i}^{n-1} \psi_{n-1}(t)+\cdots+u_{i}^{n-m} \psi_{n-m}(t)
$$

That is, the value at time $t \in\left(t_{n-1}, t_{n}\right]$ depends only on those at a time no later than $t_{n}$ and no earlier than $t_{n-m}$. In terms of the $\tau$-normalized basis functions $\Psi_{\ell}(\tau)$ the expansion in the above is

$$
\sum_{j=0}^{N_{t}} u_{i}^{j} \psi_{j}(t)=u_{i}^{n} \Psi_{0}\left(\frac{t-t_{n}}{\Delta t}\right)+u_{i}^{n-1} \Psi_{-1}\left(\frac{t-t_{n-1}}{\Delta t}\right)+\cdots+u_{i}^{n-m} \Psi_{-m}\left(\frac{t-t_{n-m}}{\Delta t}\right)
$$

Equation (13) shows that a use of the basis function of the form (10) results in an interpolation for the value at $t \in\left(t_{n-1}, t_{n}\right]$ from those on temporal nodal points $t_{n-m}, \cdots, t_{n-1}, t_{n}$.

\subsection{Generalized temporal basis function}

To further analyze the accuracy of an interpolation scheme of the form (14), we consider a general interpolation problem for a value at $\tilde{t}_{j}$, where $\tilde{t}_{j}=t_{j}-\eta \Delta t, 0 \leq \eta<1$, using a stencil from $t_{j-M}$ to $t_{j+N}$ :

$$
U\left(\tilde{t}_{j}\right)=\sum_{\ell=-M}^{N} S_{\ell}(\eta) u^{j+\ell}
$$

as illustrated in Fig. 2. Here $U(t)$ denotes the approximation for $u(t)$ by the interpolation. To satisfy causality for the interpolation, it would be required that $N=0$. The analysis presented below, however, will be valid for any $N \geq 0$. For brevity, the subscript in $u_{i}^{j}$ has been dropped in (15).

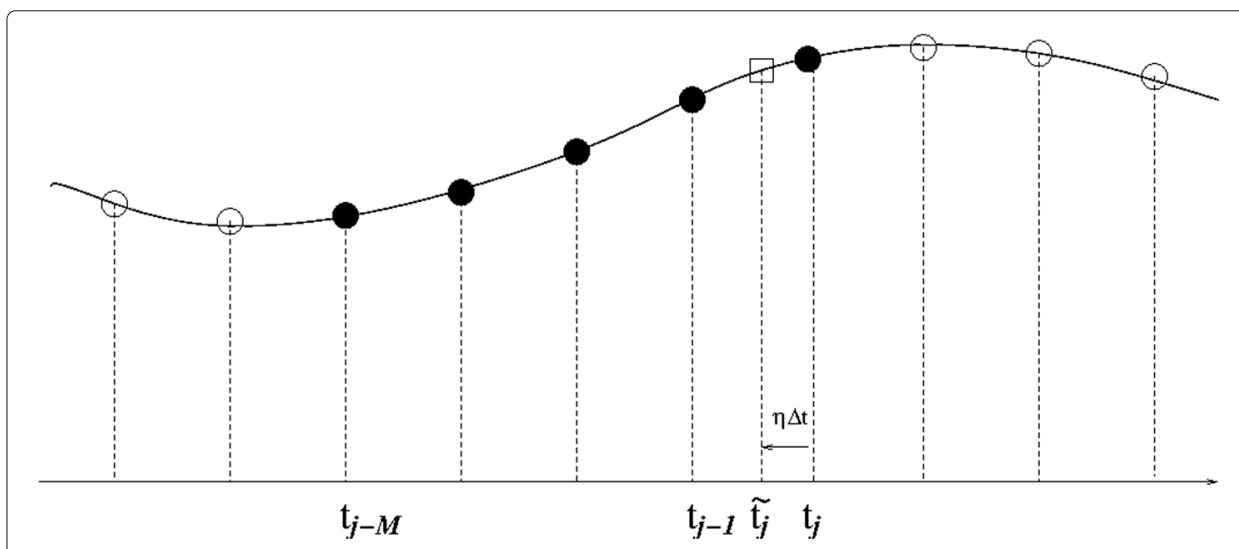

Fig. 2 A schematic diagram for a general interpolation stencil (denoted by closed circles) along the time grid for a point $\tilde{t}_{j}$ (denoted by the open square) between $t_{j-1}$ and $t_{j}$. Open circles indicate the temporal grid points 
In (15), $S_{\ell}(\eta)$ are the coefficients of the interpolation scheme for the value at any point $\tilde{t}_{j}$ located in $\left(t_{j-1}, t_{j}\right]$. For the Lagrange interpolation polynomials, we have simply

$$
S_{\ell}(\eta)=\frac{\prod_{\ell^{\prime}=-M, \ell^{\prime} \neq \ell}^{N}\left(\tilde{t}_{j}-t_{j+\ell^{\prime}}\right)}{\prod_{\ell^{\prime}=-M, \ell^{\prime} \neq \ell}^{N}\left(t_{j+\ell}-t_{j+\ell^{\prime}}\right)}=\frac{\prod_{\ell^{\prime}=-M, \ell^{\prime} \neq \ell}^{N}\left(\ell^{\prime}+\eta\right)}{\prod_{\ell^{\prime}=-M, \ell^{\prime} \neq \ell}^{N}\left(\ell^{\prime}-\ell\right)}
$$

It is also straightforward to find that when $S_{\ell}(\eta)$ in (15) is converted to temporal basis functions and expressed similarly to that in (10), we have the equivalent $\tau$-normalized basis functions as

$$
\Psi_{\ell}(\tau)=S_{\ell}(-\tau-\ell), \quad \ell=-M, \ldots, N
$$

where $\tau$ and $\eta$ are related as

$$
\tau=\frac{\tilde{t}_{j}-t_{j+\ell}}{\Delta t}=-\eta-\ell
$$

Conversely, we have

$$
S_{\ell}(\eta)=\Psi_{\ell}(-\eta-\ell)
$$

For convenience of discussion, the Lagrange interpolation basis function as defined by (15) and (16) will be denoted as the Lag- $(M, N)$ scheme. For instance, the third-order shifted Lagrange basis function (12), with $M=3$ and $N=0$, is denoted as Lag-(3,0) scheme. The explicit forms of the $\tau$-normalized basis functions for Lag- $(4,0), \operatorname{Lag}-(5,0)$, and Lag- $(6,0)$ schemes are given in Appendix 1.

As noted earlier, in the formulation of time domain integral equations, interpolations for the derivatives with respect to time (up to second-order when the Burton-Miller type formulation is used) are also required. Their approximations can now be written as

$$
\begin{aligned}
& \frac{d U}{d t}\left(\tilde{t}_{j}\right)=-\frac{1}{\Delta t} \sum_{\ell=-M}^{N} S_{\ell}^{\prime}(\eta) u^{j+\ell} \\
& \frac{d^{2} U}{d t^{2}}\left(\tilde{t}_{j}\right)=\frac{1}{\Delta t^{2}} \sum_{\ell=-M}^{N} S_{\ell}^{\prime \prime}(\eta) u^{j+\ell}
\end{aligned}
$$

where $S_{\ell}^{\prime}(\eta)$ and $S_{\ell}^{\prime \prime}(\eta)$ are respectively the first and second derivatives of $S_{\ell}(\eta)$ with respect to $\eta$.

\section{Fourier analysis of temporal basis functions}

Next, we present an error analysis of temporal interpolation schemes (15), (20) and (21) in the Fourier frequency space. While the accuracy of an interpolation scheme is traditionally assessed by the order of the interpolating polynomials, the present error analysis will be carried out in the Fourier frequency space.

Given a grid function $u^{j}$, defined on a uniform grid of $t_{j}=t_{0}+j \Delta t$, its discrete Fourier transform is [20]

$$
\hat{u}(\omega)=\Delta t \sum_{j=-\infty}^{\infty} u^{j} e^{i t_{j} \omega}
$$


where $\omega$ is the frequency. The inverse transform for (22) is

$$
u^{j}=\frac{1}{2 \pi} \int_{-\pi / \Delta t}^{\pi / \Delta t} e^{-i t_{j} \omega} \hat{u}(\omega) d \omega
$$

Equations (22) and (23) indicate that the frequency range for the spectrum of a grid function $w^{j}$ would be one that is continuous but limited to $\frac{\pi}{\Delta t}$, the Nyquist limit. The objective of the present study is to investigate interpolation schemes that preserve the spectrum $\hat{u}(\omega)$ as much as possible.

It is well-known that a special interpolation function called cardinal interpolation, denoted as $U^{I}(t)$, that recovers the full spectrum $\hat{u}(\omega)$ as defined in (22) and (23) can be constructed as follows [20],

$$
U^{I}(t)=\frac{1}{2 \pi} \int_{-\pi / \Delta t}^{\pi / \Delta t} e^{-i t \omega} \hat{u}(\omega) d \omega=\sum_{j=-\infty}^{\infty} u^{j} \operatorname{sinc}\left(\frac{t-t_{j}}{\Delta t} \pi\right)
$$

Its Fourier spectrum is

$$
\hat{U}^{I}(\omega)=\int_{-\infty}^{\infty} U^{I}(t) e^{i \omega t} d t= \begin{cases}\hat{u}(\omega), & |\omega \Delta t|<\pi \\ 0, & |\omega \Delta t|>\pi\end{cases}
$$

While $\hat{U}^{I}(\omega)$ does preserve the full spectrum of $u^{j}$, its interpolation stencil is unfortunately infinitely wide.

We now consider the accuracy of the interpolation defined in (15), (20) and (21) in the Fourier frequency space. Following the definition of discrete Fourier transform in (22), the spectrum of the interpolated values $U\left(\tilde{t}_{j}\right)$ computed on the grid $\tilde{t}_{j}=t_{j}-\eta \Delta t$ is

$$
\begin{aligned}
\hat{U}(\eta, \omega) & =\Delta t \sum_{j=-\infty}^{\infty} U\left(t_{j}-\eta \Delta t\right) e^{i\left(t_{j}-\eta \Delta t\right) \omega}=\Delta t \sum_{j=-\infty}^{\infty}\left(\sum_{j=-M}^{N} S_{\ell}(\eta) u^{j+\ell}\right) e^{i\left(t_{j}-\eta \Delta t\right) \omega} \\
& \left.\left.=\sum_{\ell=-M}^{N} S_{\ell}(\eta)\left(\Delta t \sum_{j=-\infty}^{\infty} u^{j+\ell} e^{i t_{j} \omega}\right) e^{-i \eta \omega \Delta t}\right)\right] \\
& =e^{-i \eta \omega \Delta t} \sum_{\ell=-M}^{N}\left[S_{\ell}(\eta) e^{-i \ell \omega \Delta t}\left(\Delta t \sum_{j=-\infty}^{\infty} u^{j+\ell} e^{i t_{j+\ell} \omega}\right)\right] \\
& =e^{-i \eta \omega \Delta t}\left(\sum_{\ell=-M}^{N} S_{\ell}(\eta) e^{-i \ell \omega \Delta t}\right)
\end{aligned}
$$

As the last expression in the above shows, an interpolation scheme of the form given in (15) will modify the spectrum of the original data $u^{j}$ by a factor of

$$
F_{0}(\eta, \xi)=e^{-i \eta \xi}\left(\sum_{\ell=-M}^{N} S_{\ell}(\eta) e^{-i \ell \xi}\right)
$$

where $\xi$ is the non-dimensional frequency,

$$
\xi=\omega \Delta t
$$

The ideal value for $F_{0}(\eta, \xi)$ is of course unity for all $\eta$ and $\xi$, and any deviation of $F_{0}(\eta, \xi)$ from unity represents the error of interpolation in the Fourier frequency space. 
To facilitate the ensuing discussions, we introduce the following as a measure for the error of interpolation in the frequency space:

$$
E_{0}(\eta, \xi)=\sum_{\ell=-M}^{N} S_{\ell}(\eta) e^{-i \ell \xi}-e^{i \eta \xi}
$$

Furthermore, interpolation errors for approximating the first and second derivatives with respect to time will be assessed by $E_{1}(\eta, \xi)$ and $E_{2}(\eta, \xi)$ respectively defined as follows:

$$
\begin{aligned}
& E_{1}(\eta, \xi)=\frac{d E_{0}}{d \eta}(\eta, \xi)=\sum_{\ell=-M}^{N} S_{\ell}^{\prime}(\eta) e^{-i \ell \xi}-i \xi e^{i \eta \xi} \\
& E_{2}(\eta, \xi)=\frac{d^{2} E_{0}}{d \eta^{2}}(\eta, \xi)=\sum_{\ell=-M}^{N} S_{\ell}^{\prime \prime}(\eta) e^{-i \ell \xi}+\xi^{2} e^{i \eta \xi}
\end{aligned}
$$

While convenient to work with, the error functions $E_{0}(\eta, \xi), E_{1}(\eta, \xi)$, and $E_{2}(\eta, \xi)$ can be shown to be related to the relative errors in the Fourier space for the approximations (15), (20) and (21), respectively (see Appendix 2). Clearly, magnitude of the errors depends on $\eta$ as well as $\xi$. The value $\eta$ indicates the location of the point between two nodal points and the value $\xi$ is the frequency non-dimensionalized by the time step. For instance, a value of $\xi=\pi / n$ represents a wave of period $2 n \Delta t$. For the Lagrange basis functions of order 3 to 6 , the maximum values of these errors in the range of $0 \leq \eta<1$ as functions of the non-dimensional frequency $\xi$ are plotted in Fig. 3. It is seen that for a given interpolation stencil, the largest error occurs for $E_{2}$ for interpolating the second derivatives. On the other hand, all the errors become smaller when the number of stencils increases.

As Fig. 3 reveals, the magnitude of the interpolation errors in Fourier frequency space is not the same for approximating $U, d U / d t$, and $d^{2} U / d t^{2}$, being largest for $E_{2}(\eta, \xi)$ and the smallest for $E_{0}(\eta, \xi)$. To reduce the impact of the lowered accuracy in approximating the derivatives, it is proposed that a hybrid approach be taken where a wider stencil be used for computing $d U / d t$ and $d^{2} U / d t^{2}$. For instances, when Lag- $(3,0)$ is used for the expansion for $U$, Lag- $(4,0)$ or Lag- $(5,0)$ might be used for the expansion for $d U / d t$ and $d^{2} U / d t^{2}$ in (20) and (21). In this way, the accuracy in spectrum approximation can
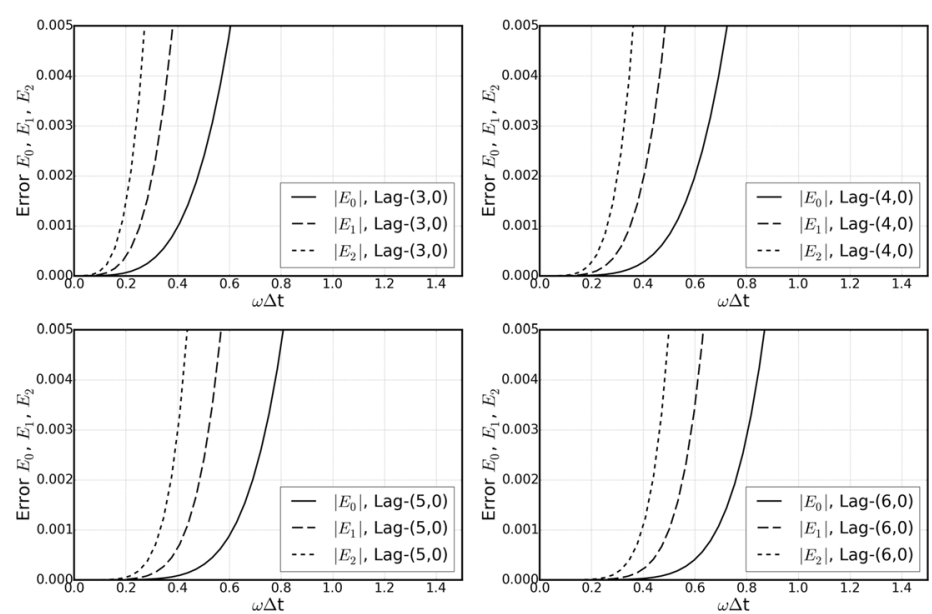

Fig. 3 Maximum of error functions $E_{0}(\eta, \xi), E_{1}(\eta, \xi)$ and $E_{2}(\eta, \xi)$ for $0 \leq \eta<1$ as a function of $\xi=\omega \Delta t$, for Lagrange schemes Lag-(3,0), Lag(4,0), Lag-(5,0), and Lag- $(6,0)$ as indicated 
be kept relatively the same. Temporal schemes with one set of basis functions for $U$ and another for its derivatives will be referred to as hybrid schemes, denoted by Lag- $(M, N)$ $\left(M_{1}, N_{1}\right)-\left(M_{2}, N_{2}\right)$ where $\left(M_{1}, N_{1}\right)$ and $\left(M_{2}, N_{2}\right)$ indicate, respectively, the interpolation stencils used for approximating the first and second derivatives. To the author's best knowledge, this is the first time a hybrid temporal basis function is proposed for MOT formulations.

Of particular interest would be the hybrid scheme Lag-(3,0)-(5,0)-(6,0). For this particular combination, the errors curves in Fig. 3 behave similarly when $\left|E_{0}\right|$ for Lag-(3,0), $\left|E_{1}\right|$ for Lag-(5,0), and $\left|E_{2}\right|$ for Lag- $(6,0)$ are compared. All the three curves show a spectral resolution up to about $\omega \Delta t=0.6$, or about 11 points per wavelength, if a criteria of errors being less than $0.5 \%$ is used. This is a significant increase from using Lag$(3,0)$ for all three types of interpolations where the spectral resolution would be limited to about 25 points per wavelength. Unfortunately, Lag- $(3,0)-(5,0)-(6,0)$ hybrid scheme is found to be unstable by the eigenvalues analysis to be presented in Section 6. In fact, the only stable hybrid schemes using the Lagrange basis functions are Lag- $(3,0)-(4,0)$ $(4,0)$ and Lag- $(3,0)-(4,0)-(5,0)$ when Lag- $(3,0)$ is used for approximating $U$. To gain still a broader spectral resolution and better stability properties, we consider in the next section optimized temporal basis function to be coupled with Lag-(3,0).

\section{Optimized scheme}

We carry out an optimization of the Lagrange interpolation scheme where the formal order of interpolation is lowered but spectral resolution is increased. The general polynomial form of $S_{\ell}(\eta)$ for an interpolation scheme written in (15) is:

$$
S_{\ell}(\eta)=\sum_{n=0}^{M+N} a_{n}^{(\ell)} \eta^{n}
$$

The coefficients $a_{n}^{(\ell)}$ are to be determined such that the following expression, which is a sum of the three types of error functions in the Fourier space,

$$
\text { OBJECTIVE : } \quad E=\int_{-\xi_{0}}^{\xi_{0}} \int_{0}^{1}\left[\left|E_{0}(\eta, \xi)\right|^{2}+\left|E_{1}(\eta, \xi)\right|^{2}+\left|E_{2}(\eta, \xi)\right|^{2}\right] d \eta d \xi
$$

is minimized. In (33), $E_{0}(\eta, \xi), E_{1}(\eta, \xi)$ and $E_{2}(\eta, \xi)$ are that given in (29), (30) and (31) respectively, and $\xi_{0}$ is a chosen limit of the non-dimensional frequency for optimization.

In carrying out the optimization process, two additional conditions will also be imposed. The first is the order of interpolation $m$. By expanding (29) as a Taylor series in $\xi$ and enforcing the equation up to order $m$, we get

$$
\sum_{\ell=-M}^{N} S_{\ell}(\eta) \ell^{k}-(-\eta)^{k}=0, \quad k=0,1,2, \ldots, m
$$

This condition ensures that the resulting optimized interpolation is accurate to order $m$ in $\eta$ and $\xi$. In terms of the coefficients $a_{n}^{(\ell)}$ as defined in (32), the order condition (34) leads to the following constraint for optimization: 


$$
\text { CONSTRAINT I : } \quad \sum_{\ell=-M}^{N} \ell^{k} a_{n}^{(\ell)}=(-1)^{k} \delta_{n k}, \quad n=0,1, \ldots, M+N, \quad k=0,1, \ldots, m
$$

In particular, when the highest possible order for (34) is enforced at $m=M+N$, Lagrange interpolation results. For the optimizations carried out in this section, the imposed order will be taken to be less than $M+N$, which frees up some of the coefficients $a_{n}^{(\ell)}$ for optimization.

The second condition is to ensure that for the point that falls on the grid, $\tilde{t}_{j}=t_{j}$, the interpolation will return exactly the value at the grid point, i.e., by (15), we require that

$$
U\left(t_{j}\right)=\sum_{\ell=-M}^{N} S_{\ell}(0) u^{j+\ell}=u^{j}
$$

This leads to

$$
\text { CONSTRAINT II : } \quad a_{0}^{(0)}=1, \text { and } a_{0}^{(\ell)}=0, \quad \ell \neq 0
$$

To find stable hybrid schemes where Lag- $(3,0)$ is used for approximating $U(t)$, minimization of (33) has been carried out for the case of $M=5$ and $N=0$, by lowering the formal order to be $m=3$ in (34) and letting $\xi_{0}=0.5$ in (33). The optimized scheme will be denoted as Opt- $(5,0)$ and is given below:

$\tau$-normalized optimized Opt- $(5,0)$ basis functions:

$$
\Psi(\tau)=\left\{\begin{array}{cc}
1+2.253708396282178 \tau+1.802980567846060 \tau^{2} & -1<\tau \leq 0 \\
+0.6435629579203238 \tau^{3}+0.09800825706055226 \tau^{4}+0.003717475566444615 \tau^{5} & \\
1.000000022219608+1.065309832128350 \tau-0.6500315444685530 \tau^{2} & 0<\tau \leq 1 \\
-1.031571801034928 \tau^{3}-1.031571801034928 \tau^{4}-0.02170250467191372 \tau^{5} & \\
1.044537108372981+0.1359348949708500 \tau-0.9227689658981300 \tau^{2} & 1<\tau \leq 2 \\
-0.7129692500148930 \tau^{3}+0.4056309092908941 \tau^{4}+0.04963526302320873 \tau^{5} & \\
0.04191437020961000+1.961226842612910 \tau-3.482150290025250 \tau^{2} & 2<\tau \leq 3 \\
+1.518893756288150 \tau^{3}-0.02495127344286200 \tau^{4}-0.05 .586551670259000 \tau^{5} & \\
4.045886482021400-6.017025386585420 \tau+2.570200374862790 \tau^{2} & 3<\tau \leq 4 \\
+0.009592652006509000 \tau^{3}-0.2090154495994932 \tau^{4}+0.03104788519098566 \tau^{5} & \\
-1.096201066066790+0.2270301998286800 \tau+0.6817698576829370 \tau^{2} & 4<\tau \leq 5 \\
-0.4275083151651400 \tau^{3}+0.09233156086346440 \tau^{4}-0.006832602406135260 \tau^{5} & \\
0 & \text { other }
\end{array}\right.
$$

The errors in Fourier frequency space for the optimized Opt- $(5,0)$ scheme is shown in Fig. 4. Also shown in Fig. 4 is the error function $E_{0}$ of scheme Lag-(3,0) for approximating $U(t)$, which is seen to be now comparable with that of $E_{1}$ and $E_{2}$ of the Opt- $(5,0)$ scheme. As such, using Opt- $(5,0)$ to approximate $d U / d t$ and $d^{2} U / d t^{2}$ will result in a more uniform accuracy in the frequency space than using Lag- $(3,0)$ for approximating all the temporal derivatives.

This hybrid scheme will be denoted as Opt- $(3,0)-(5,0)-(5,0)$ to indicate that the interpolation for $U(t), d U / d t$, and $d^{2} U / d t^{2}$ is done by Lag-(3,0), Opt-(5,0), and Opt-(5,0) 


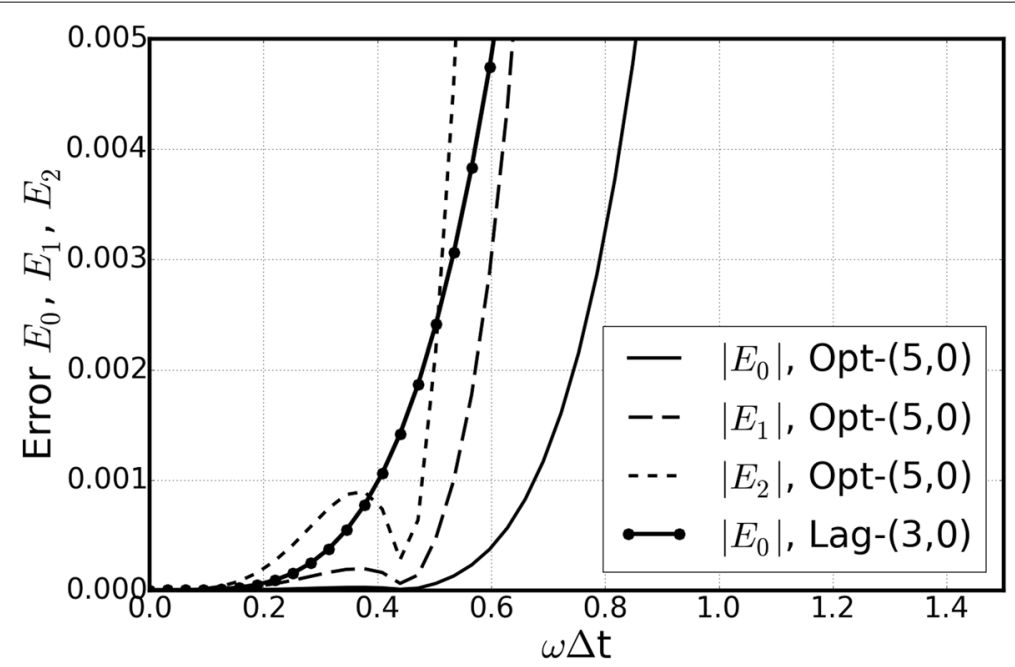

Fig. 4 Maximum of error functions $E_{0}(\eta, \xi), E_{1}(\eta, \xi)$ and $E_{2}(\eta, \xi)$ for the optimized Opt- $(5,0)$ scheme. Also shown is the error function $E_{0}$ for the Lag-(3,0) scheme

respectively. The hybrid Opt-(3,0)-(5,0)-(5,0) scheme is found to be stable by an eigenvalue analysis that is to be described in the next section.

\section{Stability of hybrid temporal schemes}

To check for the stability of the proposed hybrid schemes, a matrix eigenvalue analysis is conducted. Stability of iteration schemes of the form (6) can be analyzed by the eigenvalues of its corresponding homogeneous system [21]. We look for solutions of the form

$$
\boldsymbol{u}^{n}=\lambda^{n} \boldsymbol{e}_{0}
$$

to the homogeneous system of (6), where $\lambda$ is the amplification factor of the eigensolution. By substituting (39) into (6), we obtain a polynomial eigenvalue problem

$$
\left[\boldsymbol{B}_{0} \lambda^{K}+\boldsymbol{B}_{1} \lambda^{K-1}+\boldsymbol{B}_{2} \lambda^{K-2}+\cdots+\boldsymbol{B}_{K-1} \lambda+\boldsymbol{B}_{K}\right] \boldsymbol{e}_{0}=0
$$

The above can be cast into a generalized eigenvalue problem as follows:

$$
\left[\begin{array}{cccccc}
-\boldsymbol{B}_{1} & -\boldsymbol{B}_{2} & \cdots & \cdots & -\boldsymbol{B}_{K-1} & -\boldsymbol{B}_{K} \\
\boldsymbol{I} & 0 & \cdots & \cdots & 0 & 0 \\
0 & \boldsymbol{I} & \cdots & \cdots & 0 & 0 \\
\cdots & \cdots & \cdots & \cdots & \cdots & \cdots \\
0 & 0 & \cdots & \cdots & 0 & 0 \\
0 & 0 & \cdots & \cdots & \boldsymbol{I} & 0
\end{array}\right]\left[\begin{array}{c}
\boldsymbol{e}_{K-1} \\
\boldsymbol{e}_{K-2} \\
\cdot \\
\cdot \\
\boldsymbol{e}_{1} \\
\boldsymbol{e}_{0}
\end{array}\right]=\lambda\left[\begin{array}{cccccc}
\boldsymbol{B}_{0} & 0 & 0 & \cdots & 0 & 0 \\
0 & \boldsymbol{I} & 0 & \cdots & 0 & 0 \\
0 & 0 & \boldsymbol{I} & \cdots & 0 & 0 \\
\cdots & \cdots & \cdots & \cdots & \cdots & \cdots \\
0 & 0 & 0 & \cdots & \boldsymbol{I} & 0 \\
0 & 0 & 0 & \cdots & 0 & \boldsymbol{I}
\end{array}\right]\left[\begin{array}{c}
\boldsymbol{e}_{K-1} \\
\boldsymbol{e}_{K-2} \\
\cdot \\
\cdot \\
\boldsymbol{e}_{1} \\
\boldsymbol{e}_{0}
\end{array}\right]
$$

where $\boldsymbol{e}_{j}=\lambda^{j} \boldsymbol{e}_{0}$. For numerical scheme (6) to be stable, it is required that $|\lambda| \leq 1$ for all eigenvalues of (41).

Eigenvalues of the generalized eigenvalue problem (41) can be found via a sparse eigenvalue solver available in MATLAB and Python. Since here the eigenvalue analysis is conducted numerically, the tests for stability of the hybrid schemes have been carried out for two types of geometry. One is a smooth body of a sphere with radius being 0.5 and the other a sectional convex parabolic wing defined as follows:

$$
z=0.1 L_{x}\left(1-x^{2} / L_{x}^{2}\right), \quad-L_{x} \leq x \leq L_{x}, \quad-L_{y} \leq y \leq L_{y}
$$


where $L_{x}=L_{y}=0.5$. The scattering surfaces of the sphere and parabolic wing are discretized, respectively, by 960 and 1092 quadrilateral elements. It is found that stable hybrid schemes are Lag-(3,0)-(4,0)-(4,0), Lag- $(3,0)-(4,0)-(5,0)$, as well as Opt- $(3,0)-(5,0)$ $(5,0)$ as noted in Table 1.

\section{Numerical results}

In this section, the hybrid temporal basis functions are applied to the numerical solution of scattering of a point source by a sphere, as shown in Fig. 5 . The sphere is assumed to have a rigid surface for which an analytical solution exists. The radius of the sphere is 0.5 and a point source is placed at $r=(0,0,1)$. Specifically, the source term in (1) is of the form

$$
q(\boldsymbol{r}, t)=e^{-\sigma t^{2}} \delta\left(\boldsymbol{r}-\boldsymbol{r}_{0}\right)
$$

where $\boldsymbol{r}_{0}=(0,0,1)$ and $\sigma=1.42 /(6 \Delta t)^{2}$. By solving the time domain boundary integral equation, scattering solutions at all frequencies, within the resolution of time domain temporal basis functions used, can be obtained in one single time domain calculation. In Fig. 5, the time domain solution and the converted frequency domain solution are both illustrated.

To examine the accuracy and benefits of the hybrid temporal basis functions, numerical solution along a line in the plane $z=-2.5$ and $y=0$ will be compared with the exact solution, as shown in Fig. 6. For the examples presented in this section, 32,642 constant elements are used. Here, the extra fine resolution on the surface is used for the purpose of isolating as much as possible the errors by the temporal basis functions from that by the spatial discretization.

We define an L2 error of numerical solution along a field line as follows:

$$
E_{L_{2}}(\omega)=\frac{\left\|\hat{U}(\boldsymbol{r}, \omega)-\hat{u}_{\text {exact }}(\boldsymbol{r}, \omega)\right\|_{2}}{\left\|\hat{u}_{\text {exact }}(\boldsymbol{r}, \omega)\right\|_{2}}
$$

where $\hat{u}_{\text {exact }}(\boldsymbol{r}, \omega)$ is the known analytical solution. The L2 errors as defined in (43) are tabulated in Table 1 for results at frequencies $\omega \Delta t=\pi / 12, \pi / 8, \pi / 6$, and $\pi / 4$. The chosen frequencies are respectively for spectral resolution of $24,16,12$, and 8 points per wavelength.

Compared with the traditional Lag-(3,0) temporal basis function, using Lag- $(4,0)$ for the first and second derivatives in the hybrid approach increases the spectral resolution considerably. For instance, for the frequency of $\omega \Delta t=\pi / 8$, namely to resolve a wave period of $16 \Delta t$, the Lag- $(3,0)$ scheme has an error of nearly $2 \%$ while the hybrid Lag$(3,0)-(4,0)-(4,0)$ has the error reduced to less than $0.4 \%$. Furthermore, using the optimized

Table 1 A list of stable hybrid schemes where $U(t)$ is interpolated by using the Lag- $(3,0)$ scheme and

\begin{tabular}{|c|c|c|c|c|c|}
\hline Hybrid schemes denoted as & Eigenvalue & Temporal & Resolution & Errors & $($ L2 norm (43)) \\
\hline$(M, N)-\left(M_{1}, N_{1}\right)-\left(M_{2}, N_{2}\right)$ & analysis & $\omega \Delta t=\pi / 12$ & $\omega \Delta t=\pi / 8$ & $\omega \Delta t=\pi / 6$ & $\omega \Delta t=\pi / 4$ \\
\hline Lag-(3,0)-(3,0)-(3,0) & Stable & 0.00533780 & 0.01995454 & 0.04894223 & 0.15217784 \\
\hline Lag-(3,0)-(4,0)-(4,0) & Stable & 0.00091520 & 0.00389055 & 0.01211062 & 0.09690772 \\
\hline Lag-(3,0)-(4,0)-(5,0) & Stable & 0.00106346 & 0.00413456 & 0.01189096 & 0.05654576 \\
\hline Opt-(3,0)-(5,0)-(5,0) & Stable & 0.00114423 & 0.00392707 & 0.00700110 & 0.01436286 \\
\hline
\end{tabular}
its derivatives by a wider stencil

Also shown are the errors in $L 2$ norm for the example presented in Section 7 
A

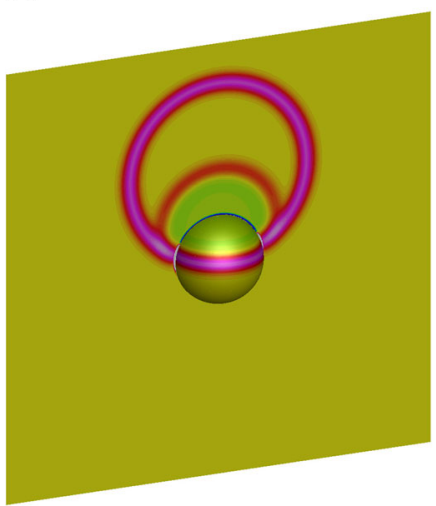

C

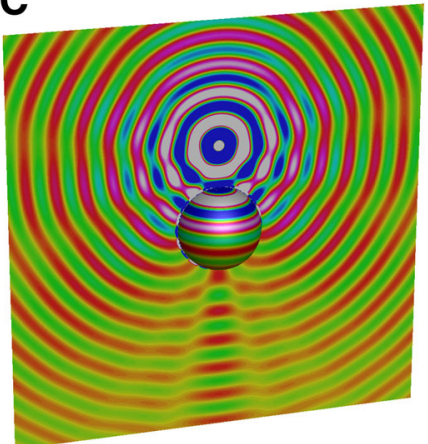

B

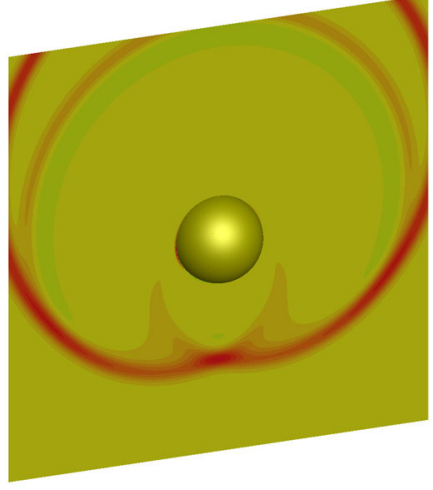

D

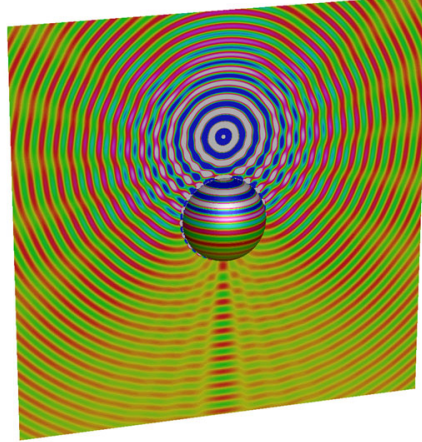

Fig. 5 Illustration of a time domain solution ( $\mathbf{a}$ and $\mathbf{b}$ ) and its converted frequency domain solution (c and $\mathbf{d}$ ), scattering of a point source at point $(0,0,1)$ by a sphere of radius $a=0.5$ centered at $(0,0,0)$. Shown are the solutions at frequency $\omega \Delta t=\pi / 8$ and $\pi / 4$, computed with $c \Delta t / a=1 / 24$

hybrid scheme Opt-(3,0)-(5,0)-(5,0), the solution at $\omega \Delta t=\pi / 6$, or a wave of period $12 \Delta t$, is still quite accurate with an error of $0.7 \%$. Solutions along a field line located at $\boldsymbol{r}=$ $(x, 0,-2.5)$ for $-2.5 \leq x \leq 2.5$ are shown in Fig. 6 . By using the optimized Opt-(3,0)$(5,0)-(5,0)$ scheme, even the solution at $\omega \Delta t=\pi / 4$, or a wave of period $8 \Delta t$, is reasonably accurate while the traditional Lag- $(3,0)$ scheme, on the other hand, has lost its resolution completely.
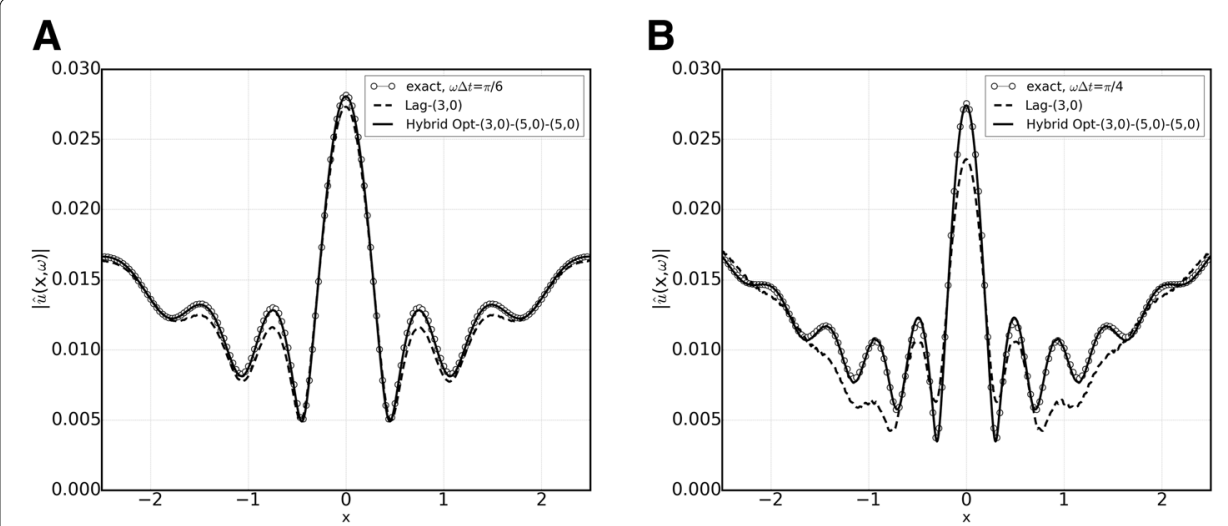

Fig. 6 Frequency domain solution along a field line $\boldsymbol{r}=(x, 0,-2.5)$ for $-2.5 \leq x \leq 2.5$. Shown are the computational results by using the traditional Lag- $(3,0)$ and the hybrid scheme Opt- $(3,0)-(5,0)-(5,0)$ schemes. Also shown is the analytic solution for comparison. (a): $\omega \Delta t=\pi / 6 ;(\mathbf{b}): \omega \Delta t=\pi / 4$ 
For the solution of time domain integral equations, an increase in time step also results in a reduction in memory requirement. For the MOT scheme shown in Eq. (6), storage of the solution up to the previous $K$ time steps are required where $K$ is the following [8]:

$$
K=\frac{L}{c \Delta t}+M
$$

in which $L$ is the spatial dimension of the scattering body and $M$ is the limit of interpolation stencil appearing in (15). So $K$ is nearly inversely proportional to the time step $\Delta t$. An increase in resolution from 16 points per wavelength to 8 points per wavelength, for instance, results in a reduction of memory by nearly $50 \%$.

\section{Conclusions}

A Fourier analysis of the temporal basis functions used in the March-On-in-Time schemes is presented. One of the advantages of a Fourier analysis is that it provides a quantitative measure of the temporal interpolation errors in the frequency space. Temporal resolutions of 3rd, 4-th, 5-th, and 6-th shifted Lagrange basis functions are presented. Based on the formulation of the Fourier analysis, hybrid temporal basis functions that significantly extend the resolution in the frequency space are proposed. Stability of the hybrid scheme is achieved by an optimization process that reduces the formal order of the scheme but extends the spectral solution. The substantial gain in accuracy as well as stability of the new basis functions are demonstrated by numerical examples. The use of hybrid basis functions keeps the error low for a larger range in the frequency space and, conversely, for a given range of frequency of interest, a larger time step can be used with the optimized temporal basis functions. The increased time step size not only results in an increase in computational efficiency, it reduces substantially the requirement on the memory as well.

\section{Appendix 1}

The classical Lagrange $\tau$-normalized basis functions for the 4th-, 5th-, and 6th-order are listed in this appendix. Also listed are the optimized Opt- $(3,0)$ and Opt- $(4,0)$ schemes. The Opt- $(5,0)$ scheme is given in (38).

Lag-(4,0), 4th-order basis functions:

$$
\Psi(\tau)=\left\{\begin{array}{cc}
1+\frac{25}{12} \tau+\frac{35}{24} \tau^{2}+\frac{5}{12} \tau^{3}+\frac{1}{24} \tau^{4} & -1<\tau \leq 0 \\
1+\frac{5}{6} \tau-\frac{5}{6} \tau^{2}-\frac{5}{6} \tau^{3}-\frac{1}{6} \tau^{4} & 0<\tau \leq 1 \\
1-\frac{5}{4} \tau^{2}+\frac{1}{4} \tau^{4} & 1<\tau \leq 2 \\
1-\frac{5}{6} \tau-\frac{5}{6} \tau^{2}+\frac{5}{6} \tau^{3}-\frac{1}{6} \tau^{4} & 2<\tau \leq 3 \\
1-\frac{25}{12} \tau+\frac{35}{24} \tau^{2}-\frac{5}{12} \tau^{3}+\frac{1}{24} \tau^{4} & 3<\tau \leq 4 \\
0 & \text { other }
\end{array}\right.
$$


Lag-(5,0), 5th-order basis functions:

$$
\Psi(\tau)=\left\{\begin{array}{cl}
1+\frac{137}{60} \tau+\frac{15}{8} \tau^{2}+\frac{17}{24} \tau^{3}+\frac{1}{8} \tau^{4}+\frac{1}{120} \tau^{5} & -1<\tau \leq 0 \\
1+\frac{13}{12} \tau-\frac{5}{8} \tau^{2}-\frac{25}{24} \tau^{3}-\frac{3}{8} \tau^{4}-\frac{1}{24} \tau^{5} & 0<\tau \leq 1 \\
1+\frac{1}{3} \tau-\frac{5}{4} \tau^{2}-\frac{5}{12} \tau^{3}+\frac{1}{4} \tau^{4}+\frac{1}{12} \tau^{5} & 1<\tau \leq 2 \\
1-\frac{1}{3} \tau-\frac{5}{4} \tau^{2}+\frac{5}{12} \tau^{3}+\frac{1}{4} \tau^{4}-\frac{1}{12} \tau^{5} & 2<\tau \leq 3 \\
1-\frac{13}{12} \tau-\frac{5}{8} \tau^{2}+\frac{25}{24} \tau^{3}-\frac{3}{8} \tau^{4}+\frac{1}{24} \tau^{5} & 3<\tau \leq 4 \\
1-\frac{137}{60} \tau+\frac{15}{8} \tau^{2}-\frac{17}{24} \tau^{3}+\frac{1}{8} \tau^{4}-\frac{1}{120} \tau^{5} & 4<\tau \leq 5 \\
0 & \text { other }
\end{array}\right.
$$

Lag-(6,0), 6th-order basis functions:

$$
\Psi(\tau)=\left\{\begin{array}{cc}
1+\frac{40}{20} \tau+\frac{203}{90} \tau^{2}+\frac{49}{48} \tau^{3}+\frac{35}{144} \tau^{4}+\frac{7}{240} \tau^{5}+\frac{1}{720} \tau^{6} & -1<\tau \leq 0 \\
1+\frac{77}{60} \tau-\frac{49}{120} \tau^{2}-\frac{7}{6} \tau^{3}-\frac{7}{12} \tau^{4}-\frac{7}{60} \tau^{5}-\frac{1}{120} \tau^{6} & 0<\tau \leq 1 \\
1+\frac{7}{12} \tau-\frac{7}{6} \tau^{2}-\frac{35}{48} \tau^{3}+\frac{7}{48} \tau^{4}+\frac{7}{48} \tau^{5}+\frac{1}{48} \tau^{6} & 1<\tau \leq 2 \\
1-\frac{49}{36} \tau^{2}+\frac{7}{18} \tau^{4}-\frac{1}{36} \tau^{5} & 2<\tau \leq 3 \\
1-\frac{7}{12} \tau-\frac{7}{6} \tau^{2}+\frac{35}{48} \tau^{3}+\frac{7}{48} \tau^{4}-\frac{7}{48} \tau^{5}+\frac{1}{48} \tau^{6} & 3<\tau \leq 4 \\
1-\frac{77}{60} \tau-\frac{49}{120} \tau^{2}+\frac{7}{6} \tau^{3}-\frac{7}{12} \tau^{4}+\frac{7}{60} \tau^{5}-\frac{1}{120} \tau^{6} & 4<\tau \leq 5 \\
1-\frac{49}{20} \tau+\frac{203}{90} \tau^{2}-\frac{49}{48} \tau^{3}+\frac{35}{144} \tau^{4}-\frac{7}{240} \tau^{5}+\frac{1}{720} \tau^{6} & 5<\tau \leq 6 \\
0 & \text { other }
\end{array}\right.
$$

Opt- $(3,0)$ basis functions (optimized using $m=2$ and $\xi_{0}=0.3$ ):

$$
\Psi(\tau)=\left\{\begin{array}{cc}
1+1.820157538622123 \tau+0.9767629249288535 \tau^{2}+0.1566531198507175 \tau^{3} & -1<\tau \leq 0 \\
1.000113218750436+0.4956014496892710 \tau-1.015054210410179 \tau^{2} & 0<\tau \leq 1 \\
-0.4806604580295282 \tau^{3} & 1<\tau \leq 2 \\
1.019228975642691-0.5462868241880500 \tau-0.9643869448304550 \tau^{2} & \\
+0.4913615565069039 \tau^{3} & 2<\tau \leq 3 \\
0.9954960870152810-1.833678754987600 \tau+1.002678230311782 \tau^{2} & \text { other }
\end{array}\right.
$$


Opt- $(4,0)$ basis functions (optimized using $m=3$ and $\xi_{0}=0.3$ ):

$$
\Psi(\tau)=\left\{\begin{array}{cc}
1+2.071420901300948 \tau+1.431748981057308 \tau^{2} & -1<\tau \leq 0 \\
+0.3968541430128426 \tau^{3}+0.03652710780440761 \tau^{4} & \\
0.9999963005105105+0.8273470830390340 \tau-0.8387509509706590 \tau^{2} & 0<\tau \leq 1 \\
-0.8381631715385190 \tau^{3}-0.1504292610403665 \tau^{4} & \\
0.9725752546323810+0.09692699551253000 \tau-1.383137253924310 \tau^{2} & 1<\tau \leq 2 \\
+0.08151469866566900 \tau^{3}+0.2321251362946538 \tau^{4} & \\
1.187102550021850-1.177122587323870 \tau-0.6000184370871300 \tau^{2} & 2<\tau \leq 3 \\
+0.7640435452328490 \tau^{3}-0.1590709206858386 \tau^{4} & \\
0.8614444096135440-1.922272308274318 \tau+1.390157660924802 \tau^{2} & 3<\tau \leq 4 \\
-0.4042492153728422 \tau^{3}+0.04084793762714370 \tau^{4} & \\
0 & \text { other }
\end{array}\right.
$$

\section{Appendix 2}

In this section, we show the relationship between the error functions $E_{0}(\eta, \xi), E_{1}(\eta, \xi)$, $E_{2}(\eta, \xi)$ defined in (29)-(31) and the approximation errors in the Fourier space.

Under the order condition (34), we have $E_{i}(\eta, \xi)=O\left(\xi^{m+1}\right)$ for $i=0,1,2$, where $\xi=\omega \Delta t$. By Eq. (21) and the definition of $E_{0}(\eta, \xi)$ in (29), we get the following relation between the Fourier spectrum of the original grid data $\hat{u}(\omega)$ and that of the interpolated values $\hat{U}(\eta, \xi)$ :

$$
\hat{U}(\eta, \xi)=e^{-i \eta \xi}\left(E_{0}(\eta, \xi)+e^{i \eta \xi}\right) \hat{u}(\omega)=\left(e^{-i \eta \xi} E_{0}(\eta, \xi)+1\right) \hat{u}(\omega)
$$

It follows that

$$
\left|\frac{\hat{U}(\eta, \xi)-\hat{u}(\omega)}{\hat{u}(\omega)}\right|=\left|E_{0}(\eta, \xi)\right|
$$

Therefore, $E_{0}(\eta, \xi)$ represents the relative error of the interpolation approximation $U\left(\tilde{t}_{j}\right)$ of $(15)$ in the Fourier space.

Similarly, using Eqs. (20) and (21) and the definition of $E_{1}(\eta, \xi)$ and $E_{2}(\eta, \xi)$ by (30) and (31), respectively, it can be shown that

$$
\left|\frac{\frac{\widehat{d u}}{d t}(\eta, \xi)-\widehat{\frac{d u}{d t}}(\omega)}{\frac{\widehat{d u}}{d t}(\omega)}\right|=\frac{\left|E_{1}(\eta, \xi)\right|}{|\xi|}
$$

and

$$
\left|\frac{\frac{d^{2} u}{d t^{2}}(\eta, \xi)-\frac{\widehat{d^{2} u}}{d t^{2}}(\omega)}{\frac{\widehat{d^{2} u}}{d t^{2}}(\omega)}\right|=\frac{\left|E_{2}(\eta, \xi)\right|}{\left|\xi^{2}\right|}
$$

where it is assumed that, for the original grid data, $\frac{\widehat{d u}}{d t}(\omega)=(-i \omega) \hat{u}(\omega)$ and $\frac{\widehat{d^{2} u}}{d t^{2}}(\omega)=$ $(-i \omega)^{2} \hat{u}(\omega)$. That is, $E_{1}(\eta, \xi)$ and $E_{2}(\eta, \xi)$ are respectively related to the relative errors of approximation (20) and (21) for the first and second derivatives in the Fourier space. 
Funding

This work was supported in part by a NASA Cooperative Agreement, NNX11Al63A.

\section{Availability of data and materials}

All data generated or analysed during this study are included in this published article.

\section{Authors' contributions}

The author read and approved the final manuscript.

\section{Competing interests}

The author has no competing interests.

\section{Publisher's Note}

Springer Nature remains neutral with regard to jurisdictional claims in published maps and institutional affiliations.

Received: 23 January 2019 Accepted: 19 February 2019

Published online: 25 March 2019

\section{References}

1. Burton A, Miller G (1971) The application of integral equation methods to the numerical solution of some exterior boundary-value problems. Proc R Soc Lond A 323(1553):201-210

2. Morino $L$ (2003) Is there a difference between aeroacoustics and aerodynamics? An aeroelastician's viewpoint. AlAA J 41(7):1209-1223

3. Chappell D, Harris P, Henwood D, Chakrabarti R (2006) A stable boundary element method for modeling transient acoustic radiation. J Acoust Soc Am 120(1):74-80

4. Costabel M (2004) Time-dependent problems with the boundary integral equation method. Encycl Comput Mech $1: 703-721$

5. Ergin A, Shanker B, Michielssen E (1999) Analysis of transient wave scattering from rigid bodies using a burton-miller approach. J Acoust Soc Am 106(5):2396-2404

6. Ha-Duong T, Ludwig B, Terrasse I (2003) A Galerkin BEM for transient acoustic scattering by an absorbing obstacle. Int J Numer Methods Eng 57(13):1845-1882

7. Hackbusch W, Kress W, Sauter SA (2008) Sparse convolution quadrature for time domain boundary integral formulations of the wave equation. IMA J Numer Anal 29(1):158-179

8. Hu FQ, Pizzo ME, Nark DM (2017) On a time domain boundary integral equation formulation for acoustic scattering by rigid bodies in uniform mean flow. J Acoust Soc Am 142(6):3624-3636

9. Lubich C (1994) On the multistep time discretization of linear initial-boundary value problems and their boundary integral equations. Numer Math 67(3):365-389

10. Lubich C (1988) Convolution quadrature and discretized operational calculus. I Numer Math 52(2):129-145

11. Ergin A, Shanker B, Michielssen E (2000) Fast analysis of transient acoustic wave scattering from rigid bodies using the multilevel plane wave time domain algorithm. J Acoust Soc Am 107(3):1168-1178

12. Hu FQ (2013) An efficient solution of time domain boundary integral equations for acoustic scattering and its acceleration by graphics processing units. In: 19th AIAA/CEAS Aeroacoustics Conference. AlAA paper 2013-2018

13. Meng J, Boag A, Lomakin V, Michielssen E (2010) A multilevel cartesian non-uniform grid time domain algorithm. J Comput Phys 229(22):8430-8444

14. Thirard C, Parot JM (2017) On a way to save memory when solving time domain boundary integral equations for acoustic and vibroacoustic applications. J Comput Phys 348:744-753

15. Weile DS, Pisharody G, Chen NW, Shanker B, Michielssen E (2004) A novel scheme for the solution of the time-domain integral equations of electromagnetics. IEEE Trans Antennas Propag 52(1):283-295

16. vant Wout $\mathrm{E}$, van der Heul DR, van der Ven $H$, Vuik C (2013) Design of temporal basis functions for time domain integral equation methods with predefined accuracy and smoothness. IEEE Trans Antennas Propag 61 (1):271-280

17. Wang X, Weile DS (2011) Implicit Runge-Kutta methods for the discretization of time domain integral equations. IEEE Trans Antennas Propag 59(12):4651-4663

18. Geranmayeh A, Ackermann W, Weiland T (2009) Temporal discretization choices for stable boundary element methods in electromagnetic scattering problems. Appl Numer Math 59(11):2751-2773

19. vant Wout $E$, van der Heul DR, van der Ven H, Vuik C (2016) Stability analysis of the marching-on-in-time boundary element method for electromagnetics. J Comput Appl Math 294:358-371

20. Strikwerda JC (2004) Finite difference schemes and partial differential equations, SIAM, Philadelphia, PA

21. Dodson S, Walker S, Bluck M (1998) Implicitness and stability of time domain integral equation scattering analyses. Appl Comput Electromagn Soc J 13:291-301 